\title{
Der Bau
}

\section{Betrachtungen zu einer Metapher der Luhmannschen Systemtheorie}

\author{
Jens Soentgen \\ Eschenweg 16a, D-6370 Oberursel
}

Zusammenfassung: Luhmann verwendet als Emblem seiner Theorie oft das Bild vom labyrinthischen Bau. In diesem Aufsatz sollen einige Expeditionen in die Bilderwelt unternommen werden, auf die wir durch diese Metapher verwiesen werden. Als Anregung dienen dabei die Geschichten „Der Bau“ und „Von den Gleichnissen“ von Franz Kafka. Ziel ist es, die oft übersehene oder für unwichtig erklärte Hintergrundmetaphorik der Systemtheorie herauszupräparieren und zu zeigen, daß sich in ihr der Stimmungsgehalt der Theorie verdichtet.

\section{Einleitung}

In diesem Aufsatz möchte ich eine Metapher beschreiben, die sehr häufig auftaucht, wenn Niklas Luhmann von seiner Theorie im ganzen und von seinem Verhältnis zu ihr spricht. Es ist die Metapher des labyrinthischen Baus, ein traditionsreiches, uraltes Bild, das, wie ich zeigen werde, bei Luhmann eine charakteristische Bedeutung erhält. Eine Untersuchung dieses Bildes wird auf einfache Weise klar machen, um welche Art Gedankengebilde es sich bei dieser Supertheorie handelt, und welche Erkenntnisse man von einer Beschäftigung mit ihr erwarten kann.

Zunächst werde ich die Entsprechung, die zwischen der Baumetapher und der Luhmannschen Systemtheorie besteht, anhand einiger persönlicher Erfahrungen, die ich beim Einarbeiten in die Theorie gemacht habe, beschreiben. Dann möchte ich mich einer genaueren Untersuchung des Metaphernfeldes, das durch das Wort „Bau“ bezeichnet wird, zuwenden. Dabei dient mir als Leitfaden die Geschichte „Der Bau“ von Franz Kafka, eine wahre Fundgrube für Nachbarmetaphern dieses zentralen Bildes. Das dort aufgefundene Bildermaterial läßt sich hervorragend mit Theoriebereichen der Luhmannschen Soziologie in Verbindung bringen, wie ich an zwei Beispielen vorführen werde. Der Leser ist also eingeladen, mit mir gemeinsam in jenem Album zu blättern, aus dem Luhmann das Bild vom Bau entnommen hat, um es zum Keim seiner Theorie werden zu lassen. Es wird sich zeigen, daß in diesem Album noch viele weitere Bilder verborgen sind, die wie Vorbilder für bestimmte Konstruktionen der Theorie sozialer Systeme aussehen, oder auch wie ein ironischer Kommentar zu diesen. Ich hoffe, daß bei dieser Bildbetrachtung etwas von dem Stimmungsgehalt der Theorie, auf den Luhmann selbst einmal hingewiesen hat (Luhmann 1981b: 177), sichtbar wird.

\section{Erste Erkundungsgänge ins Innere der Theorie}

„Um praktische Vertrautheit mit Theorien, eigenen oder fremden, zu erlangen, darf man sich nicht nur an die großen, einladenden Portale halten, durch die jedermann eintreten kann. Bei weiterem Vordringen stößt man auf andersartige, auch funktionale Einrichtungen, die der Stabilisierung des Ganzen, der Verteidigung der Errungenschaften oder der Erleichterung interner Beweglichkeit und Einfallsproduktion dienen. Da gibt es Dunkelkammern, in denen man erst nach längerer Eingewöhnung etwas sieht. Nicht selten ist das der Ort, an dem der Theoretiker seine inneren Erfolge hatte und von dem aus er sich in seiner Konstruktion sicher fühlen kann. Und man ahnt, daß es $\mathrm{Ge}$ heimgänge geben müsse, die die Insassen rascher als den Kritiker zu neuen Argumenten führen, findet Scheintüren, an denen man sich vergebens $a b-$ müht, und richtige Türen, die sofort wieder nach draußen führen. Die Darstellung der Theorie vermittelt keine ausreichende Vorstellung ihres Konstruktionsplans, den die weitere Arbeit zugrundezulegen hat."

Dieser Textabschnitt aus dem Aufsatz „Die Praxis der Theorie" (Luhmann 1970: 264) gibt einen guten Einblick in die Bilderwelt, die sich Luhmann immer wieder aufzudrängen scheint, wenn er versucht, sich seine Theorie als Ganze vorzustellen. Er schildert sie als einen verwinkelten, komplizierten Bau, in dem es eine Vielzahl von Gängen und Querverbindungen gibt. Das Besondere dabei ist:

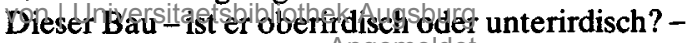


hat keinen Turm, ${ }^{1}$ von dem aus man etwa eine Landschaft überblicken könnte. Die Theorie scheint nicht dazu gebaut, einen Aus- oder Überblick zu ermöglichen, sie ragt nicht himmelwärts, sie bietet keine Orientierungshilfe. Im Gegenteil. Zunächst sieht es so aus, als sei die Konstruktion einzig dazu gedacht, den Eintretenden zu verwirren: Scheintüren, an denen man sich vergebens abmüht, richtige Türen, die sofort wieder nach drauBen führen . . . Dem entspricht, daß Luhmann offenbar ein ambivalentes Verhältnis zur Publizität, zur allgemeinen Zugänglichkeit seiner Theorie hat. Einerseits ist die Theorie natürlich veröffentlicht, jeder kann sie lesen. Andererseits aber liebt es der Autor, den Leser irrezuführen, ihm bewußt zweideutige Formulierungen zuzumuten, an denen er sich ,abzumühen" hat.

Nur ein Beispiel aus eigener Erfahrung: Was bedeutet der Satz ,nur die Kommunikation kann kommunizieren" (Luhmann 1990: 31)? Es mag an der Dummheit liegen, mit der ich gestraft bin, aber mir ist es erst nach langem, heftigem Nachdenken gelungen, mir auf diese Formel einen Reim zu machen. Wie soll man sich das Kommunizieren der Kommunikation vorstellen: Stimmen aus dem Nichts? Ich habe es mir schließlich so zurechtgelegt, daß der Satz nicht wörtlich zu nehmen ist, sondern eine Art Parodie darstellt auf den ähnlich lautenden Satz „Nur der Mensch kann kommunizieren“. Dieser Satz erscheint uns zwar einleuchtender als der mit der Kommunikation, er ist aber ebenso absurd, weil natürlich ein Mensch allein auch nicht kommunizieren könnte. Wenn aber diese Interpretation zutrifft (und nicht ,sofort wieder nach draußen führt"), dann fragt man sich: hätte man das nicht auch einfacher sagen können?

1 Es ist mir bekannt, daß Luhmann einmal, nämlich in "Autopoiesis als soziologischer Begriff" (im Diskussionsband von Haferkamp/Schmid 1987: 308) von einer Art Turmspitze, die seine Theorie enthalte, gesprochen hat. Er bezeichnet dort als "Zinne“ unter vielen "Zacken“ der Theorie die Selbstreferenz. Ich halte aber die Verwendung dieser Metapher für einen Lapsus, sie paßt überhaupt nicht zu seiner Theorie. An vielen Stellen sagt ja Luhmann explizit, da $B$ diese gerade nicht das Ziel hat, eine privilegierte Beobachtungsposition aufzubauen. Das wird aber durch die Metapher vom Gebirge und von den Gipfels suggeriert. Vermutlich ist der Fehlgriff, der Luhmann sonst, so weit ich sehen kann, nicht mehr unterlaufen ist, erklärbar durch den suggestiven Zauber der Alliteration von „Zacken“ und ,Zinnen“, sowie durch die Gelegenheit, hier einmal ein Zitat eines von ihm geschätzten Dichters, Höldeîlinęiejasutel| bringen.
Nehmen wir jetzt einmal an, wir hätten die groBen, trügerischen Portale gemieden und einen der verborgenen Eingänge in die Theorie entdeckt. Dann hat man tatsächlich den Eindruck, sich im Innern eines weitläufigen, labyrinthischen Gangsystems zu befinden. Zumindest passen die Erfahrungen, die ich beim Lesen von Luhmanns Büchern gemacht habe, sehr gut zu diesem Bild. Oft verfolgte ich einen Gang, ein bestimmtes Thema, bog um eine Ecke und befand mich plötzlich auf einem Platz, den ich kannte, den ich hier aber gar nicht erwartet hätte. So las ich einmal in „Liebe in Passion“ über die Entwicklung der „Liebessemantik“, und plötzlich, auf S. 218 führten die Argumente auf ein ganz anderes Terrain, auf das Thema „abnehmende Bedeutung der Religion in der modernen Gesellschaft". Und dann, als sei nichts geschehen, gelangte ich wieder auf den Hauptgang. So kam es, daß ich oft nach und nach völlig die Orientierung verlor. Hinter der nächsten Ecke, auf der nächsten Seite konnte ja alles mögliche kommen. Oft wußte ich nicht mehr, wo ich mich befand, manchmal fand ich nicht einmal mehr zum Ausgang zurück. Dann kreisten meine Gedanken stundenlang um einzelne Sätze, oder ich versuchte den Plan des Baus, in den ich geraten war, zu entschlüsseln. Oft bin ich über solchen Grübeleien eingeschlafen. Irgendwie schien es mir im Schlaf leichter zu fallen, den Ausgang zu entdecken, denn beim Erwachen fand ich mich stets zurückversetzt in mein natürliches, unkompliziertes Zuhause, in dem die Menschen wieder kommunizieren können, in dem Liebe ein Gefühl ist, und in dem man wieder „ißt, um satt zu werden“. ${ }^{2}$

Soviel zu meinen persönlichen Erfahrungen im Labyrinth. Viele Luhmann-Leser werden ähnliche gemacht haben. Wenn man eine Weile lang Erkundungsgänge durch den Bau gemacht hat, stellt sich angesichts der Ausdehnung des Komplexes - immer wieder stößt man auf Gänge, die man noch nie betreten hat, und die Expansion schreitet fort die Frage: Was ist der Sinn dieser ungeheuren Anstrengung? Wenn es sich bei der Theorie ja um einen riesenhaften, babylonischen Turm handeln würde, wenn die Arbeit darauf zielte, eine konkurrenzlose Beobachtungsposition aufzubauen, dann könnte man ihren Sinn ohne weiteres einsehen; aber der Luhmannsche Bau geht nicht in die Höhe, sondern in die Tiefe und in die Breite - welchen Zweck soll dieses Bauwerk, dieser „Schacht

2 Von Luhmann für den Alltagsgebrauch ausdrücklich

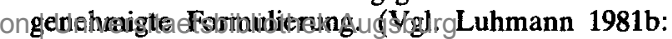

44).

Angemeldet

Heruntergeladen am | 19.09.19 11:29 
von Babel“" (Kafka 1986: 280) haben? Luhmann gibt auf diese Frage eine lakonische Antwort: „Die Theorie behauptet, ihr eigenes Ziel zu sein. (. . .) Sie ist, was sie ist. Sie ist ein ,Selbstzweck'." (Luhmann 1987b: 39)

Mit einer solchen Antwort wird sich natürlich kein Leser zufriedengeben. Stattdessen werden seine Erkundungsgänge nicht selten von einer Idee motiviert sein, die von der Metapher vom unterirdischen Bau beinahe zwingend nahegelegt wird: Man stellt sich vor, daß ohne Zweifel am Ende des letzten Gangs ein Schatz gelagert ist. Wenn es so teuflisch schwer ist, sich in diese Theorie einzuarbeiten, dann muB in ihrer letzten Kammer auch mindestens der Stein der Weisen liegen. Was soviel Anstrengung kostet, muß auch die Mühe wert sein: gerade die dunkelsten Höhlen geben Anla zu den gewagtesten Hoffnungen. Je weiter man im Labyrinth vordringt, je mehr Arbeit man daran wendet, desto mehr verdichtet sich die Gewißheit, die große Entdeckung müsse unmittelbar bevorstehen. Man wünscht sich, hier den Schlüssel zu finden, der einem alle unverständlichen Erfahrungen, die man in dieser Gesellschaft gemacht hat, mit einem Male erklärt.

Auch meine Erkundungsgänge wurden lange von einer solchen Hoffnung geleitet. Ich kann nur sagen, da $B$ ich bislang nicht fündig geworden bin. Bis auf einige kleinere Lebensweisheiten, etwa die, $\mathrm{daB}$, an allem Geschehen immer System und Umwelt beteiligt sind", daß man also doch nicht der Alleinurheber seiner Taten ist, bin ich auf nichts lebensweltlich Brauchbares gestoßen.

Nach einer Weile habe ich dann von der Schatzsuchermentalität abgelassen und bin zu einem „Kenner und Schätzer von Bauten“ (Kafka 1961: $390)^{3}$ geworden. Ich begann, die Eleganz des Baus zu bewundern. Und allmählich wandelte sich die anfängliche Unsicherheit in seinem Innern in ein Gefühl der Vertrautheit und Sicherheit. Etwas ähnliches muß auch Luhmann empfinden, der sich in "seiner Konstruktion sicher fühlt" (Luhmann 1970: 264). Das Bild vom Gangsystem hängt eng zusammen mit dem Bild vom Versteck: im Labyrinth können sich die Angreifer verlaufen, der Bewohner wird immer überlegen sein. Diese Erfahrung scheinen auch die Kritiker gemacht zu haben, die sich 1986 mit Luhmann trafen, um über den Text „Soziale Systeme“ zu diskutieren. Sie hatten den Eindruck, daß es in dem Bau immer eine Art

3 Der Baumeister in Kafkas Erzählung würde schimpfen: „ein wüster Lump, der wohnen will, ohne zu bauen." (Kafka 1961: 390) uneinnehmbaren Burghof gibt, auf den sich „die Theorie in Zeiten der Bedrängnis . . zurückzieht" (Haferkamp/Schmid 1987: 14). Das also scheint der eigentliche Sinn des Baus zu sein: Schutz zu bieten gegen mögliche Angriffe jeder Art.

Ich möchte nun den erwähnten Text „Der Bau“ von Kafka untersuchen, in dem dieses Motiv - der Bau als Schutzraum gegen Feinde - besonders hervorgehoben wird. Es wird sich dabei zeigen, daß Kafkas Geschichte eine wahre Fundgrube von Nachbarmetaphern darstellt, die in der Nähe des "Urbildes“, vom Bau gelagert sind, und die bei Luhmann beständig durchscheinen, ohne immer, wie im Fall des Labyrinths, direkt benannt zu werden. Der Baumeister in Kafkas Geschichte äußert sich oft mit fast den gleichen Worten über sein Werk und die Probleme, die er mit ihm hat, wie Luhmann. Es ist, als hätte der Jurist Kafka in dieser Gestalt und ihrem „Heim“ den Juristen Luhmann und seine Systemtheorie vorausgeahnt. Ein längeres Zitat mag einen Eindruck von der Verwandtschaft der Bilderwelt im Hintergrund der Systemtheorie und jenen Bildern, die die Geschichte „Der Bau“ tragen, vermitteln.

\section{Der Bau}

Ich habe den Bau eingerichtet und er scheint wohlgelungen. Von außen ist eigentlich nur ein großes Loch sichtbar, dieses führt aber in Wirklichkeit nirgends hin, schon nach ein paar Schritten stößt man auf natürliches festes Gestein. Ich will mich nicht dessen rühmen, diese List mit Absicht ausgeführt zu haben, es war vielmehr der Rest eines der vielen vergeblichen Bauversuche, aber schließlich schien es mir vorteilhaft, dieses eine Loch unverschüttet zu lassen. . . . Wohl tausend Schritte von diesem Loch entfernt liegt . . . der eigentlich $\mathrm{Zu}$ gang zum Bau ..." (Kafka 1961: 378). „Ich habe dort ein volles Zickzackwerk von Gängen angelegt, dort fing mein Bau an, ich durfte damals noch nicht hoffen, ihn je so beenden zu können, wie er in meinem Plane dastand, ich begann halb spielerisch an diesem Eckchen und so tobte sich dort die erste Arbeitsfreude in einem Labyrinthbau aus, der mir damals die Krone aller Bauten schien, den ich aber heute wahrscheinlich richtiger als allzu kleinliche, des Gesamtbaus nicht recht würdige Bastelei beurteile, die zwar theoretisch köstlich ist - hier ist der Eingang zu meinem Haus, sagte ich damals ironisch zu den unsichtbaren Feinden und

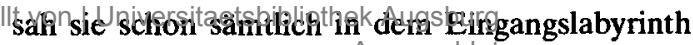


ersticken -, in Wirklichkeit aber eine viel zu dünnwandige Spielerei darstellt ..." (Kafka 1961: 384f).

Um die 35 Seiten lange, unvollendete Erzählung, die von Kafka im Herbst 1923 geschrieben wurde, kurz zusammenzufassen: Der Ich-Erzähler, anscheinend ein großes, fleischfressendes Waldtier, schildert zunächst die Architektur seines weitläufigen und komplexen, unterirdischen Baus. Die Motivation zur Anlage des Gangsystems liegt hier zum einen im Schutz gegen Feinde; zum anderen ist es aber auch die Freude an der Raffinesse des Baus, die die Mühe seiner Anlage lohnt. Dann beschreibt der Baumeister die Abenteuer und Ängste eines Ausflugs nach draußen. Wieder zurückgekehrt, bemerkt er ein leises Zischen, das plötzlich in den Gängen auftaucht. Es gelingt ihm nicht, die Ursache ausfindig zu machen. Schließlich gerät er in Panik und wähnt sich eingekreist von den Gängen eines riesigen, ungeheuer starken Tieres, das ohne Pause, nach einem geheimnisvollen, gigantischen Plan gräbt. Bald, so fürchtet er, wird dieser andere, mächtiger Gräber auf seinen Bau stoßen, ihn darin auffinden und vernichten. Mit diesen Befürchtungen endet die Geschichte. ${ }^{4}$

Ich werde nun zwei der Motive nachgehen, die in dem Text auftauchen, und zu zeigen versuchen, daß diese auch im Hintergrund vieler Äußerungen Luhmanns aufleuchten, und viele scheinbar begriffliche, rationale Äußerungen auf charakteristische Weise „einfärben“.

\section{Die Waldmäuse}

Man hat sich oft darüber aufgeregt, daß Luhmann eine gewisse Arroganz gegenüber empirischen Soziologen an den Tag legt. Darüber beklagt sich lautstark etwa Haferkamp in dem zitierten Diskussionsband „... . empirische Forschung (wird von Luhmann zwar) pauschal gelobt, aber für weitreichende Theorie für unzureichend erklärt." (Haferkamp/Schmid 1986: 57). Und wirklich räumt Luhmann selbst zwar ein, daß die Soziologie ,als empirische Wissenschaft den Anspruch nicht aufgeben (kann), ihre Aussagen an Hand von Daten zu überprüfen, die der Realität abgewonnen sind“ (Luhmann 1988: 7), belächelt aber andererseits die empirischen Sozialforscher, die nichts anderes betrieben als „die Exegese selbstproduzierter Da-

4 Es existieren allerdings Mutmaßungen über die von Kafka geplante Fortsetzung. Siehe Politzer 1978: 506. ten" (Luhmann 1987c: 5). Dennoch ist seine Theorie keineswegs empirielos. Vielmehr gelingt es ihm immer wieder, die Ergebnisse empirischer Untersuchungen sehr geschickt mit Hilfe seiner Theorie $\mathrm{zu}$ interpretieren. Doch haben diese Untersuchungen auf der Abstraktionsebene, auf der sich Luhmann bewegt, nie den Status einer Verifikationsoder Falsifikationsinstanz, und das ist es wahrscheinlich, was die Kritiker stört. In der Theorie sozialer Systeme hat die Empirie eindeutig eine untergeordnete Rolle. Vorgefundenes empirisches Material wird der Tendenz nach assimiliert, wenn es paßt, und distanziert, wenn es nicht zu verwerten ist. ${ }^{5}$ Die Theorie ist souverän im Umgang mit dem, was empirische Untersuchungen zu Tage fördern. Sie räumt solchen Untersuchungen an keiner Stelle die Möglichkeit ein, ihre Integrität zu verletzen. Die Theoriedynamik der Systemtheorie wird zentral von internen Entwicklungserfordernissen determiniert. Oder anders gesagt: daß die Theorie Erfahrungskontakt benötigt, steht zwar außer Frage, aber das "Wie" ihres Erfahrungsbezuges bestimmt sie selbst.

Das Waldtier aus Kafkas Erzählung bringt dieses schwierige Verhältnis zur Empirie und zu empirischen Sozialforschern auf den Punkt: „. . . mit der Außenwelt (verbinden mich) noch ganz enge, ziemlich ungefährliche Wege, die mir gut atembare Luft verschaffen. Sie sind von den Waldmäusen angelegt. Ich habe es verstanden, sie in meinen Bau richtig einzubeziehen. Sie bieten mir auch die Möglichkeit ausreichender Witterung und geben mir so Schutz." (Kafka 1961: 379-380). An anderer Stelle spricht es statt von Waldmäusen vom „Kleinzeug“, und klagt: „Viel Zeit, viel Zeit, die besser verwendet werden könnte, kostet mich das kleine Volk." Halb verwundert, halb verächtlich stellt es fest, daß das Kleinzeug planlos gräbt, „ohne Sinn ... oder nur, weil es die Erde frißt." (Kafka 1961: 403).

Was den Baumeister an den Aktivitäten der Waldmäuse besonders stört, ist, daß diese bisweilen Gänge bohren, die die feinbalancierte Ventilation aus dem Gleichgewicht bringen, wodurch ein $\mathrm{Zi}$ schen entsteht, das die absolute Stille im Innern des Baus ruiniert. Dieses Ereignis tritt ein in der Mitte der Geschichte. Aus einem tiefen Schlaf wird das Tier geweckt durch ,ein an sich kaum hörbares Zischen . . . Ich verstehe sofort, das Kleinzeug, viel zu wenig von mir beaufsichtigt, viel zu

5 Den Gründen dafür können wir hier nicht nachgehen einige Andeutungen finden sich z. B. in Luhmann $1987 \mathrm{~b}$. 
sehr von mir geschont, hat in meiner Abwesenheit irgendwo einen neuen Weg gebohrt, dieser Weg ist mit einem alten zusammengestoßen, die Luft verfängt sich dort, und das ergibt das zischende $\mathrm{Ge}$ räusch. Was für ein unaufhörlich tätiges Volk das ist und wie lästig sein Fleiß! Ich werde, genau horchend, an den Wänden meines Ganges, durch Versuchsgrabungen den Ort der Störungen erst feststellen müssen und dann erst das Geräusch beseitigen können. Übrigens kann der neue Graben, wenn er irgendwie den Verhältnissen des Baus entspricht, als neue Luftzuführung mir auch willkommen sein. Aber auf die Kleinen will ich nun viel besser achten als bisher, keines darf geschont werden.“ (Kafka 1961: 397). „Bei solchen Gelegenheiten ist es gewöhnlich das technische Problem, das mich lockt, ich stelle mir zum Beispiel nach dem Geräusch, das mein Ohr in allen seinen Feinheiten zu unterscheiden die Eignung hat, . . . die Veranlassung vor, und nun drängt es mich, nachzuprüfen, ob die Wirklichkeit dem entspricht." (Kafka 1961: 398)

Ganz ähnlich geht Luhmann in dem Buch „Ökologische Kommunikation" den Irritationen nach, denen seine Theorie durch die alarmierenden empirischen Untersuchungen zum Umweltproblem ausgesetzt ist. Auch hier beginnen die Nachforschungen mit herablassenden Bemerkungen über die wissenschaftliche Relevanz der Bohrungen des Kleinzeugs, sprich: der empirischen Datenproduzenten. ${ }^{6}$ Deren rastlose, dabei aber völlig planlose Arbeit setzt Luhmann in Erstaunen; ihre Resultate werden so beurteilt: „Die Unbekümmertheit in der Wortwahl und das mangelnde Gespür für folgenreiche Theorieentscheidungen sind eines der auffälligsten Merkmale dieser Literatur - so als ob die Sorge um die Umwelt die Sorglosigkeit der Rede darüber rechtfertigen könnte." (Luhmann 1986: 8). Trotzdem muß Luhmann feststellen, daß sich die Ergebnisse als ,nicht länger ignorierbares, störendes ,Rauschen“" in der Gesellschaft bemerkbar machen. Damit ist aber auch die Ruhe seines Labyrinths, welches ja als Nachbau dieser Gesellschaft angelegt ist, gestört. So macht er sich denn auf, die Ursache des Zischens zu ermitteln.

Dabei geht er genauso vor, wie sein Doppelgänger in Kafkas Geschichte: ausgehend vom Zentrum (den Kernbegriffen: Komplexität, Evolution, Beobachtung, Kommunikation, System, Codierung) schreitet er seine Theorie ab bis zu den äußersten, schon recht umweltnahen Gängen (den Konzepten für Wirtschaft, Recht, Politik usw.). Überall stellt er das gleiche Geräusch („Resonanz") fest.

Die Irritation wird also nicht zum AnlaB genommen, die Theorie zu verlassen, um etwa nachzusehen, was da draußen vor sich geht - oder gar einzugreifen! - sondern es geht um „Theorietechnik“, um eine Untersuchung, wie sich das Umweltproblem auf die Theorie sozialer Systeme auswirkt: es geht ausschließlich darum, „zu sehen, welche Konturen das Problem annimmt, wenn man es mit Hilfe dieser Theorie formuliert." (Luhmann 1986: 25).

Um besser hören zu können, legt Luhmann sogar einige neue Gänge in seiner Theorie an, bzw. baut alte um. Es ist oft erstaunlich, welche Freiheit sich der Baumeister gegenüber seiner eigenen Theorie bewahrt, mit welcher Souveränität er neue Konzepte einführt und alten eine neue Deutung gibt. Ich denke hier vor allem an die Unterscheidung zwischen Codes und Programmen und an die Diskussion von Paradoxien und Tautologien. Diese neuen Konzepte werden mit großer Sorgfalt in den bestehenden theoretischen Komplex eingepaßt: die Veränderungen sollen unsichtbar bleiben. ${ }^{\text {? }}$

Das Kafkasche Waldwesen macht es ähnlich: „Ich werde in der Richtung zum Geräusch hin einen regelrechten großen Graben bauen und nicht früher zu graben aufhören, bis ich, unabhängig von allen Theorien, die wirkliche Ursache des Geräusches finde." Und auch dieser Gräber legt Wert darauf, stets alles in guter Ordnung zurückzulassen, die neuen Wände hinter sich zu glätten und zu plätten, eine Arbeit, sagt er stolz, ,die ich . . . unübertrefflich auszuführen imstande bin." (Kafka 1961: 404).

In beiden Fällen kommt die Suche nach der Ursache des Geräuschs zu keinem eigentlichen Abschluß. Bei Kafka bricht die Beschreibung des Suchprozesses einfach ab, die Geschichte bleibt Fragment. Ein wissenschaftlicher Autor kann diese Form des Abschlusses natürlich nicht wählen, und so rechtfertigt Luhmann die Ergebnislosigkeit seines Suchprozesses in einem eigenen Kapitel „Ursachen und Verantwortungen?" (Luhmann 1986: 26ff) als notwendig, und meint, daß auch das genaue Formulieren eines unlösbaren Problems schon interessant und lehrreich genug ist.

7 Dieses Vorgehen führt dazu, daß der nachvollziehende Leser oft das Gefühl hat, daß sich hier doch etwas verändert hat, aber selten in der Lage ist, den Ort der Veranderung genau anzugeben.
6 Dieses entfachte den Zorn des W.L. (W.(a)L.(dmaus)?) Bühl, der daraufhin Luhmann in seiner Rezension (Bühl 1987) leeres Theoretisieren, Spiegelfechterei usw. vorwarf. 


\section{Sprachverwirrung}

Luhmann arbeitet daran, aus seiner Theorie eine „,selbsttragende Konstruktion“ (Luhmann 1988: 11) zu machen. In diese Richtung zielen seine Bemühungen, eine "theorieeigene Sprache“ (Luhmann 1981a: 170) zu entwickeln, die von der Alltagssprache abgekoppelt ist. Durch Verwendung im Theoriekontext erhalten einige Begriffe eine neue Bedeutung, die sich aus der Verknüpfung mit anderen theorieeigenen Vokabeln ergibt. Diese Verknüpfung ist zirkulär angelegt: „die Begriffe sollen sich, soweit möglich, aneinander schärfen" (Luhmann 1988: 12). ${ }^{8}$ So soll ein ausdifferenziertes Sprachgebilde entstehen, das eine eigene Semantik und eine eigene Weltsicht konstituiert.

Wie muß man sich solch ein geschlossenes Sprachgebilde im Endstadium vorstellen, und wie würde es in der Kommunikationspraxis funktionieren? Schon jetzt zeichnen sich große Verständigungsprobleme zwischen Luhmann und seinen Kollegen ab, die zunehmen werden, je weiter die Entwicklung des Projektes ,theorieeigene Sprache“ getrieben wird. ${ }^{9}$ Anscheinend führt auch ein „Schachtbau zu Bielefeld" zur Sprachverwirrung, jedenfalls im Kleinen. Je mehr Begriffe von Luhmann abweichend definiert werden, desto geringer wird die Anzahl der Gemeinsamkeiten, die bei Diskussionen zur Klärung von Mißverständnissen herangezogen werden können. Extrapoliert man diese Entwicklung, so kommt als Grenzfall ein absurder Dialog in den Blick: beide Seiten reden völlig aneinander vorbei, und Mißverständnisse können nicht mehr behoben werden, da es keine Gemeinsamkeiten mehr gibt, auf die man zu diesem Zweck zurückgreifen könnte.

Ein Modell einer solchen Sprachverwirrung findet sich in Kafkas Geschichte "Von den Gleichnissen“ (Kafka 1961: 328):

„Viele beklagen sich, daß die Worte der Weisen immer wieder nur Gleichnisse seien, aber unver-

8 Die neue Bedeutung der alten Begriffe muß man lange trainieren. Woran denken Sie z. B. bei dem Wort „Penetration“? Fällt Ihnen da gleich die Definition ein: „Von Penetration wollen wir sprechen, wenn ein System die eigene Komplexität . . . zum Aufbau eines anderen Systems zur Verfügung stellt" (Luhmann 1988: 290)? Mir fällt dabei immer der Biologieunterricht in der 6 . Klasse ein, wo auch oft von „Penetration“ die Rede war, allerdings in ganz anderen Zusammenhängen.

9 Einen ersten Eindruck davon vermitteln einige Interviews in Luhmann 1987a. Vgl. besonders $14-37,58-$ 60, 99-107. wendbar im täglichen Leben, und nur dieses allein haben wir. Wenn der Weise sagt: „Gehe hinüber“, so meint er nicht, daß man auf die andere Seite hinübergehen solle, was man immerhin noch leisten könnte, wenn das Ergebnis des Weges wert wäre, sondern er meint irgendein sagenhaftes Drüben, etwas, das wir nicht kennen, das auch von ihm nicht näher zu bezeichnen ist, und das uns also hier gar nichts helfen kann. Alle diese Gleichnisse wollen eigentlich nur sagen, daß das Unfaßbare unfaßbar ist, und das haben wir gewußt. Aber das, womit wir uns jeden Tag abmühen, sind andere Dinge.

Darauf sagte einer: „Warum wehrt ihr euch? Würdet ihr den Gleichnissen folgen, dann wäret ihr selbst Gleichnisse geworden und damit schon der täglichen Mühe frei."

Ein anderer sagte: „Ich wette, daß auch das ein Gleichnis ist."

\section{Der erste sagte: „Du hast gewonnen.“}

Der zweite sagte: „Aber leider nur im Gleichnis."

Der erste sagte: „Nein, in Wirklichkeit; im Gleichnis hast du verloren." (Kafka 1961: 328)

Untersuchen wir zunächst in groben Zügen die logische Grundstruktur des Textes ${ }^{10}$ (vgl. Bux 1988): In der Geschichte werden zwei Personengruppen, die Gruppe der Weisen und die Gruppe der „Pragmatiker" miteinander konfrontiert, die scheinbar dieselbe Sprache gebrauchen, sich aber unter den einzelnen Wörtern jeweils Verschiedenes vorstellen. Im ersten Teil stellt ein Mann der Praxis, dessen kleinkariertes und an Nützlichkeit orientiertes Denken stark an die Waldmäuse erinnert, die Welt der Weisen und ihre Sprache aus seiner Sicht dar. Zunächst bemerkt er, daß die Sprache der Weisen ihre eigene Semantik hat, der Satz „Gehe hinuber" hat in ihr eine andere Bedeutung als in der Umgangssprache. Dann stellt er fest, daß die Aussagen, die in dieser Sprache gemacht werden, in seinem täglichen Leben absolut unnütz sind. Dabei legt er ein pragmatisches Nützlichkeitskriterium zugrunde, wonach nur solche Sätze, die sich als strategische Handlungsanweisungen verstehen lassen, nützlich sind. Die Sätze der Weisen, die diesem Kriterium nicht genügen, erscheinen ihm daher sinnlos. Für ihn sind sie reine Tautologien. Inplizit formuliert er die Frage nach der Rechtfertigung dieser Tautologien.

10 Damit ist natürlich noch keine vollständige Interpretation geliefert. Eine solche hätte u.a. zu berücksichtigen, daß der Satz "Gehe hinüber“ auf ein apokryphes Jesus-Wort anspielt; Die Welt ist eine Brücke. Gehe hinuber, aber lab dich nicht auf ihr nieder." 
Darauf antwortet ihm nun ein Weiser: „Warum wehrt ihr euch? Würdet ihr den Gleichnissen folgen, dann wäret ihr selber Gleichnisse geworden, und damit schon der täglichen Mühe frei." Man erkennt sofort, daß der Weise die Worte nicht in ihrem eigentlichen, sondern im übertragenen Sinne gebraucht: um die Verständnisschwierigkeiten, die der Pragmatiker mit der Sprache der Weisen hat, $\mathrm{zu}$ beheben, verwendet der Weise wieder ein Gleichnis!

Entschlüsselt bedeutet dieses Gleichnis etwa folgendes: „Die Voraussetzung dafür, daß ihr den Gleichnissen einen Sinn abgewinnen könnt, ist, $\mathrm{da} \beta \mathrm{ihr}$ aufhört, alles nach dem Kriterium des direkten, handgreiflichen Nutzens zu beurteilen. (Denn Gleichnisse haben keinen unmittelbaren Nutzen.) Gelänge euch dies, so würdet ihr weise werden. Ihr könntet mit Besitz und Nutzen gelassener umgehen und wäret nicht länger deren Sklaven. Euer tägliches Leben würde sich entspannen und euch nicht mehr so anstrengen. Indirekt würden euch die Gleichnisse also auch nach eurem Kriterium nutzen. Das allerdings setzte voraus, daß ihr es zuvor aufgebt." Das Nützlichkeitskriterium bildet aber gerade das Fundament der Denkungsart der Pragmatiker! Die Forderung, es aufzugeben, zielt nicht auf einen einfachen Kompro$\mathrm{miB}$, sondern auf eine völlige Umstülpung des Weltbildes, auf eine Art Bekehrung. Es wundert nicht, daB sich der Pragmatiker zu diesem Akt nicht aufraffen kann. So bleibt für ihn der Satz des Weisen wieder nur eine Wortfolge ohne direkten, praktischen Sinn. Enttäuscht stellt er fest: "Ich wette, daß auch das (nur) ein Gleichnis ist."

Der Weise sieht, daß sein Partner die Welt immer noch auf seine alte Weise sieht, und stellt die (natürlich gleichnishafte) Diagnose: „.. . . in Wirklichkeit (hast du gewonnen); im Gleichnis (also im übertragenen Sinne) hast du verloren." Er hat nur im pragmatischen Sinne gewonnen, er hat den richtigen Tip abgegeben, ein Gewinn im übertragenen Sinne (innere Bereicherung, Befreiung von der eigenen Beschränktheit) liegt dagegen nicht vor.

„Von den Gleichnissen“ ist die konzentrierte Darstellung eines systematischen, unbehebbaren Mißverstehens, und hier ergeben sich deutliche Analogien zu den Verständigungsproblemen zwischen Luhmann und seinen Fachkollegen.

Auch die Aussagen Luhmanns werden ja oft als sinnlos und tautologisch bezeichnet (vgl. etwa Buhl 1987). Ebenso wird oft gesagt, daß die Theorie nutzlos sei, daß sie keinen Erkenntnisgevianul| abwirft. Geht man dem nach, dann zeigt sich, da $B$ hier ein Mißverständnis vorliegt: Luhmann versteht unter Worten wie Liebe, Wahrheit oder Erkenntnis einfach etwas anderes, als gemeinhin üblich (vgl. Luhmann 1981: 173). Vom Standpunkt der normalen sozio-philosophischen Sprache kann man ihm daher vorwerfen, daß er solche Wörter nur metaphorisch, gleichnishaft verwendet. Worauf Luhmann entgegnen könnte, daß sein abweichender Sprachgebrauch durchaus sinnvoll ist, vorausgesetzt, man teilt sein besonderes Erkenntnisinteresse. Er wird aber Schwierigkeiten haben, seinen Partnern sein Verständnis von Erkenntnis nahezubringen, da er zur Erläuterung wieder auf andere theorieeigene Vokabeln zurückgreifen müßte, welche für seine Partner wieder nur Gleichnisse sind. Selbst wenn er den ehrlichen Wunsch hat, sich seinen Kollegen verständlich zu machen, die zirkuläre Anlage seiner Theorie sorgt dafür, da $B$ jeder Versuch, sich auf sie zuzubewegen, ihn in einem ,strange loop“ wieder auf seinen Ausgangspunkt zurückwirft. Wenn sein Theorieprojekt einmal vollendet ist, dann wird es schwer für ihn werden, aus den Definitionskreisläufen seiner Konstruktion auszutreten. Vermutlich werden ab diesem Punkt Diskussion zwischen ihm und Nicht-Luhmannianern dem Gespräch zwischen dem Praktiker und dem Weisen äußerst ähnlich werden, sie könnten sich - frei nach Kafka - etwa so anhören:

„Herr Luhmann, viele beklagen sich, daß Sie die guten alten Termini nur noch metaphorisch verwenden, und so sind sie unverwendbar im normalen wissenschaftlichen Leben. Wenn Sie etwa sagen: mit meiner Theorie erkenne ich die Gesellschaft, dann meinen Sie mit Erkennen nicht jenes anschauliche und praxisrelevante Begreifen, um das es uns zu tun ist, sondern irgendeinen fremdartigen Prozeß, den wir nicht kennen, der auch von Ihnen nicht näher bezeichnet wird, und der uns also gar nichts helfen kann. Ihre Theorie ist für uns nutzlos, sie läßt sich einzig auf sich selbst anwenden."

Darauf entgegnet Luhmann: „Warum wehrt ihr euch? Ihr braucht doch nur meinen Erkenntnisbegriff zu akzeptieren, dann werdet ihr auch den Sinn von neuen Begriffen und abweichendem Sprachgebrauch einsehen. Erkenntnis setzt eine Terminologie voraus, die die nötige Distanz vom Gegenstand garantiert. Ihre Aufgabe ist vor allem, Vergleichsmöglichkeiten zu schaffen. Was Sie daran hindert, mich zu verstehen, ist, da $B$ Sie immer gleich wissen wollen, ob Sie dafür oder dagegen usein sollensiGietshübsedh diesessodirekte Verhältnis zu 
den Dingen aufgeben. Dann könnten Sie auch mit meinen Aussagen etwas anfangen, z.B. mit der, daß die Gesellschaft die Gesamtheit aller erreichbaren Kommunikationen ist (wobei Kommunikation = Synthese aus Mitteilung, Information und Verstehen).

„Herr Luhmann, auch dieser Definition fehlt wieder die Praxisrelevanz."

„Da haben Sie Recht.“

„Na, wenigstens geben Sie es zu. Ich habe ja immer gesagt, daß man sich mit Ihnen verständigen kann."

„Aber nicht wie Sie denken. Meiner Meinung nach hat keine wissenschaftliche Aussage per se Praxisrelevanz, sie kann sie gar nicht haben. Was ist Praxis? Für mich sind das die Systeme in der Umwelt des Wissenschaftssystems, die sich aus deren Aussagen selbst das heraussuchen, was für sie nützlich ist. Ihre Wahl ist vom Wissenschaftler aus nicht zu beeinflussen, daher ist es sinnlos, zu versuchen, praxisrelevante Aussagen herzustellen. Daraus folgt: Man muß vom Erkenntnisbegriff die Praxisrelevanz abpellen und dem Wort eine neue Bedeutung geben. Von meiner Position aus ist es also zwingend notwendig, das Wort „Erkenntnis“ nur noch in einem ,uneigentlichen“, metaphorischen Sinne zu gebrauchen."

Es ist gar nicht so unwahrscheinlich, daß sich dieser absurde Dialog einmal auf irgendeinem der nächsten Soziologentage abspielen wird. Wenn es wirklich dazu kommt, daß ein Nicht-Luhmannianer die Aussagen Luhmanns nicht mehr verstehen kann, dann wäre tatsächlich die Systemtheorie zu einem abgeschlossenen Sprachgebilde geworden, in welches man nur durch eine vollständige Umkehrung des Denkens, sei es durch eine Gehirnwäsche oder durch eine Erleuchtung, eintreten kann.

Umgekehrt wäre Luhmann der Mühe enthoben, sich mit den Kritiken, die andere an seiner Theorie äußern, zu befassen. Er kann schon jetzt wie jene Weisen zeigen, daß diese Kritiken auf Prämissen ruhen, die nicht notwendig, sondern kontingent sind, und die von ihm eben anders gesetzt werden. Die Formulierung seiner Theorie ist dadurch nicht einfacher, sondern schwieriger geworden, denn anstelle der fremden beschwert er sie mit eigenen Rechtfertigungslasten, die viel gewichtiger sind. Luhmann fordert von seiner Theorie, daß sie sich selbst erklären soll. Die Last, die er ihr damit auflädt, könnte in der Tat nicht größer sein: es ist ihre gesamte eigene Masse. Erst wenn der paradoxe Akt geschieht, und sie sich selbst hebt, ist der Plan erfüllt: Die selbsttragende Konstruktion präsentiert sich dem staunenden Publikum.

\section{Die Verheißung der Stille}

Auch der unterirdische Gräber in der Geschichte „Der Bau“ träumte lange Zeit davon, sich eine selbsttragende Konstruktion zu schaffen, mußte aber einsehen, daß die Realisation über seine Kräfte ging. Darüber ist seine Trauer groß: „Hätte ich doch wenigstens die wichtigsten Pläne meines Jünglings- und frühen Mannesalters ausgeführt, oder vielmehr, hätte ich die Kraft gehabt, sie auszuführen, denn an dem Willen hat es nicht gefehlt. Einer dieser Lieblingspläne war es gewesen, den Burgplatz loszulösen von der ihn umgebenden Erde, das heißt, seine Wände nur in einer etwa meiner Höhe entsprechenden Dicke zu belassen, dar-

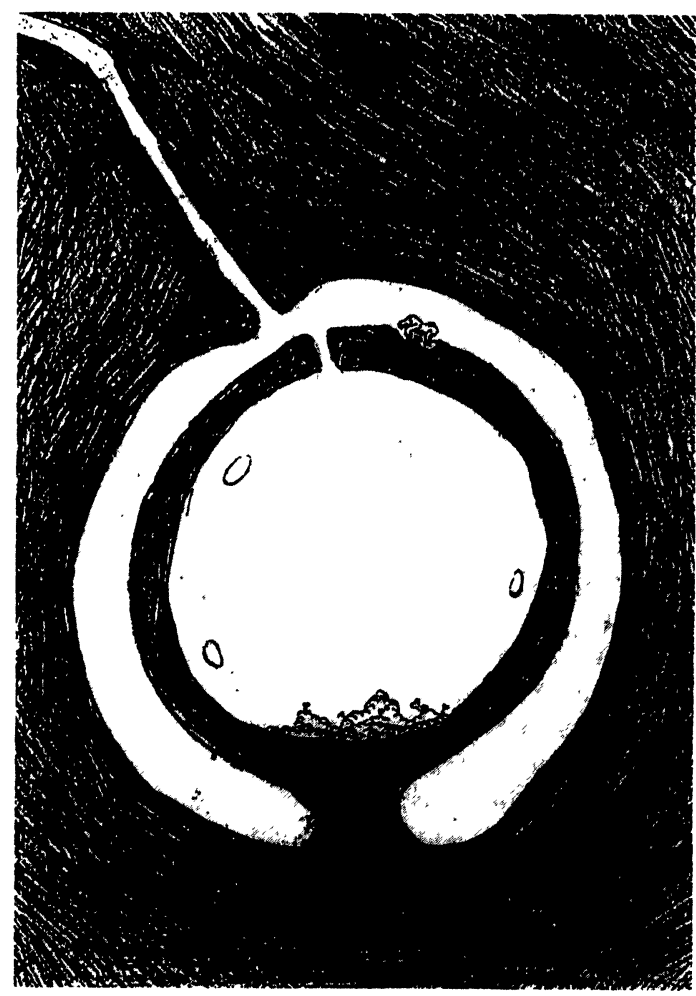

Abb. 1 Der losgelöste Burgplatz, obenauf der Baumeister, innen die Haufen erbeuteter Tiere. Vermutlich muß man sich die Konstruktion sogar noch monumentaler vorstellen. Der Burgplatz liegt in großer Tiefe, die Regi-

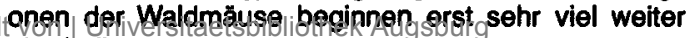
oberhalb. 
über hinaus aber rings um den Burgplatz bis auf ein kleines, von der Erde leider nicht loslösbares Fundament einen Hohlraum im Ausmaß einer Wand zu schaffen. In diesem Hohlraum hatte ich mir immer, und wohl kaum mit Unrecht, den schönsten Aufenthaltsraum vorgestellt, den es für mich geben könnte. . . . Nicht die Grabungen des kleinen Volkes hätte ich mit Widerwillen zu behorchen, sondern mit Entzücken etwas, was mir jetzt völlig entgeht: das Rauschen der Stille auf dem Burgplatz." (Kafka 1961: 400).

Das Motiv der selbsttragenden Konstruktion, das uns hier in einer ungewohnten Version begegnet, ist bekannt aus der berühmten Geschichte vom Baron Münchhausen, der sich an den eigenen Haaren aus dem Sumpf zog. In dem Moment, da es diesem gelang, sich selbst zu tragen, begann er zu fliegen, löste sich von der Erde und konnte nun wie ein Satellit alles von oben betrachten. Münchhausens wundersame Fähigkeit, sich selbst zu tragen, bescherte ihm eine privilegierte Beobachtungsposition. Das erklärt die große Faszination, die die Geschichte auf so viele Philosophen und Wissenschaftstheoretiker hat.

Kafkas Modell weicht von dieser Vorgabe gezielt ab. Zunächst bleibt der Erdbewohner im Gegensatz zu Münchhausen auf dem Boden der Tatsachen, ihm ist klar, daß sich seine Konstruktion niemals völlig von der Erde lösen ließe. Aber die Ablösung soll soweit wie möglich getrieben werden.

Der wesentliche Unterschied betrifft die Motivation: warum ist für den Baumeister eine fast selbsttragende Konstruktion so erstrebenswert? Die Verheißung von freier Beweglichkeit und freier Sicht, die in Münchhausens Geschichte implizit enthalten ist, kann für ihn schwerlich von Interesse sein. Er interessiert sich nicht für privilegierte $\mathrm{Be}$ obachtungspositionen, das reiche Panorama, das sich dem Auge in der Höhe darbietet, ist für ihn ohne Reiz. Er lebt unter der Erde, sein entwickelster Sinn ist das Gehör. Wenn er eine selbsttragende Konstruktion anstrebt, dann ist es nicht wegen der optischen, sondern wegen der akustischen Qualitäten, die diese bieten würde. Er sagt es selbst: es geht ihm um das "Rauschen der Stille“ auf dem Burgplatz. Diese akustische Sensation wünscht er zu erlangen, das ist die Verheißung, die ihn in seinen Jugend- und Mannesjahren umgetrieben hat. Der unterirdische Planet, den er formen wollte, ist innen hohl, er birgt den Burgplatz. Hier befinden sich in großen Massen erbeutete Waldmäuse, aber diese ärgern ihn nicht, da sie totosind regungslos und still. Stille: das ist es, wonach sich der Baumeister am meisten sehnt. Das Zischen, jene Folge des verdammenswerten Bohrens lebender Waldmäuse, er will es endgültig abschaffen. Seine selbsttragende Konstruktion soll ihn nicht in die Höhe heben, das konnte sie ja auch gar nicht, da sie unterirdisch ist; sie ist konzipiert als Kathedrale der Stille.

Diese Version einer selbsttragenden Konstruktion ist eine sehr viel bessere Metapher für Luhmanns Theorieprojekt als der fliegende Münchhausen. Auch Luhmann geht es nicht darum, abzuheben. Immer wieder betont er, daß keine wissenschaftliche Theorie aus der Gesellschaft austreten kann, um diese von oben zu beobachten. Als Kommunikation bleibt jede Erkenntnisbemühung umschlossen, eingeschlossen von der Gesellschaft. Sie bleibt unter der Erde. Hier gilt: „Es gibt nur Ratten im Labyrinth“ (Luhmann 1987c: 6), keine Ratte kann, durch welche Kunststücke auch immer, aus dem Labyrinth gelangen, um die Angelegenheit von außen zu beobachten.

Wohl aber kann eine Ratte versuchen, durch kunstvoll angelegte Grabungen ihre Erde von der Erde der anderen zu trennen. Das ist das Projekt einer theorieeigenen Sprache, welches Luhmann verfolgt. Wenn sein Plan realisiert ist, so erlangt er, wovon jener andere Gräber nur träumte. Er kann sich auf ein Erdreich legen, das weitestmöglich von dem der anderen losgelöst ist. So dringt das Geräusch der Waldmäuse kaum noch zu ihm. Er braucht sich nicht mehr darum zu kümmern; es ist nicht mehr seine Erde, in der jene bohren. Er kann sich ganz darauf konzentrieren, das einzigartige, paradoxe Klangphänomen, das dann zu hören sein wird, in sich einzusaugen: das ,Rauschen der Stil$1 e^{“}$. Seine Theorie genügt endlich ihren eigenen Kriterien, es gibt keine inneren Störgeräusche mehr.

\section{Wandlungen in der Erkenntnismetaphorik}

Mit seiner Metapher vom fensterlosen Bau bricht Luhmann radikal mit einer langen Metapherntradition. Frühere Theoretiker verglichen ihre Produkte etwa mit Türmen, von deren Plattform aus man Überblick und Aussicht genießen konnte, oder, sofern sie Prognosen erlaubten, mit Fernrohren, welche das raumzeitlich Entfernte nahe heranholten, oder mit Spiegeln, die es gestatten, sich selbst zu erkennen und das, was sich hinterm Rükken abspielt the Therien wurden und werden oft mit

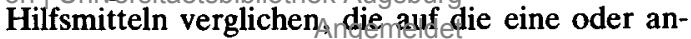


dere Weise das Blickfeld erweitern. Auch Luhmanns Metapher hat etwas mit dem Gesichtssinn zu tun, aber in genau entgegengesetzter Weise. Im Innern des Bau ist es dunkel, da gibt es nichts zu sehen. Der Blick wird nicht bereichert oder erweitert, sondern erstickt.

Dieser Umbruch in der Theoriemetaphorik impliziert einen ebenso weitreichenden Wandel in der Erkenntnismetaphorik. Der Weg der Erkenntnis führt nicht mehr, wie früher einmal, aus der Höhle heraus, sondern umgekehrt, in diese hinein ${ }^{11}$ Wenn man im Labyrinth weiter vordringt, gelangt man nicht zu einer letzten Sicherheit, im Gegenteil: die Unsicherheit wächst.

In Luhmanns Erkenntnistheorie findet sich eine begriffliche Version dieses Gedankens: Die Wissenschaft „zerstört den Halt, den man vordem an der Welt zu haben glaubte. Sie reduziert das Normale auf einen extrem unwahrscheinlichen Zufall. Sie relativiert, historisiert, exzeptionalisiert die vertrauten Bedingungen eines Menschenlebens, ohne deren Vertrautheit durch ein funktionales Äquivalent ersetzen zu können. Sie verunsichert ...". (Luhmann 1991: 654)

Es wäre freilich falsch, anzunehmen, daß es im Bau nur blindes, unsicheres Tasten gibt, und daß alles, was draußen vorgeht, von den verschachtelten Wänden weggeschluckt wird. Außenwahrnehmung gibt es sehr wohl, nur ist sie indirekt, nicht so unmittelbar wie das Sehen. Der Bau ist ein komplexer Klangkörper, ein multiresonantes System, das Geräusche auf gesetzmäßige Weise filtert und verstärkt. Hier kommt es auf das Hören an. Luhmann und das Waldtier sind Virtuosen in der Wahrnehmung und Entschlüsselung von Geräuschen. Die Baumetaphorik impliziert, daß das Gehör zum eigentlichen Tor zur Welt wird. Die verbreitete, aber falsche Meinung, daß man im Bau von der Wirklichkeit abgeschottet ist, daß seine einzige und eigentliche Funktion die der Panzerung gegen die Realität ist, mag daher rühren, daß viele auf das Sehen fixiert und ungeschult im Hören sind. Diese Wahrnehmungsform wird als anstrengend und ermüdend empfunden, und so werden die faszinierenden Laute, die im Innern des Baus zu hören

11 An prominenter Stelle, in der Einleitung zu „Soziale Systeme" vergleicht Luhmann den Gang seiner Überlegungen mit einem Flug. Aber auch hierbei soll es nichts zu sehen geben: „Der Flug muß über den Wolken stattfinden, und es ist mit einer ziemlich geschlossenen Wolkendecke zu rechnen." (Luhmann 1988: 13). sind, von vielen Eindringenden überhört oder vom Lärm ihrer irrenden Schritte übertönt.

\section{Fazit: Luhmann und Kafka}

Wie Luhmann war auch Kafka überzeugt, daß die überkommenen Vorstellungen von Wissenschaft, Kunst und Erkenntnis nicht mehr passen. Als Dichter hat er das traditionelle Metaphern- und Bildermaterial, in dem sich diese Vorstellungen ausdrücken, auf aggressivste Weise demontiert, gekippt und umgestellt. Seine Radikalität dabei ist unüberbietbar: Die Elfenbeintürme früherer Dichter rammte er ungespitzt in den Boden. So entstand der Bau, eine neue und vielseitige symbolische Formel. Mancherlei Realisationen sind denkbar für diese Formel, aber eine besonders gut passende ist sicher Luhmann und seine Soziologie. ${ }^{12}$ Und deshalb ist Kafka für mich jener Schriftsteller, nach dem Luhmann in seinem Vortrag , Unverständliche Wissenschaft" (Luhmann 1981b: 170-177) gefragt hat: ein Dichter, dessen Werk als eine literarisch-poetische Zweitfassung der systemtheoretischen Grundintuition verstanden werden kann; ein Dichter, dessen Poesie den eigentümlichen Weltstimmungsgehalt dieser ,anspruchsvollen Theorieleistung " konzentriert.

Die in der Erzählung „Der Bau“ auffallenden Parallelen sind keine zufälligen Koinzidenzen. Das literarische Werk Kafkas korrespondiert auch in anderer Hinsicht mit Luhmanns wissenschaftlicher Arbeit; insbesondere teilen beide Autoren ein starkes Interesse an Paradoxien. ${ }^{13}$ Eine Neuanalyse

12 Eine andere Möglichkeit wäre Kafka selbst und seine Texte (Vgl. für diese Interpretation Politzer 1978: 489-590). Auch Kafka war - wie Luhmann - kein Freund von einfachen, unmittelbar verständlichen Aussagen, und sein literarisches Werk wirkt auf den Eindringling ähnlich verunsichernd und ungemütlich wie Luhmanns Systemtheorie. Man könnte auch an die moderne Zivilisation als Ganze denken, diese ist ja schon oft mit einem Labyrinth verglichen worden. Es ist sicher sinnlos, zu fragen, welche Interpretation nun die richtige ist. Kafkas Poesie ist abstrakt; wie für eine mathematische Struktur gibt es für sie meist mehrere mögliche Deutungen.

13 Beispiele: in der Geschichte „Das Schweigen der Sirenen" (Kafka 1961: 300f) geht es um die Paradoxie des eingeschlossenen ausgeschlossenen Dritten; die paradoxen Beziehungen zwischen Erblindung und Erkenntnis, von Luhmann häufig thematisiert, sind ein Nebenmotiv der Erzăhlung "Vor dem Gesetz". Die Texte Kafkas haben die unangenehme Eigenart, einen zu zwingen, in das Paradox einzutreten, wogegen man bei Luhmann immer in sicherer Entfernung bleibt. 
der Texte Kafkas mit Blick auf Luhmann würde sicher noch viele Bilder ans Tageslicht befördern, die den Weltstimmungsgehalt der Luhmannschen Soziologie verdichten.

Aber andererseits werden sich natürlich, wie nicht anders zu erwarten, auch Bestandteile auf beiden Seiten finden, die sich nicht verknüpfen lassen: die Überlappung ist nur partiell. Auch findet sich Kafka meines Wissens nicht unter den zahllosen Autoren, die Luhmann zitiert. Die Übereinstimmungen lassen sich also nicht durch irgendeine „Beeinflussung“ erklären, sie scheinen eher auf etwas Drittes zu verweisen: die Gesellschaft, die beide beobachten. Kafka würde es so ausdrücken: „Das hat er nicht von mir. Das liegt in der Zeit. Wir haben es beide von ihr abgeschrieben." (Kafka in Janouch 1981: 36f).

\section{Literatur}

Bux, K.-U., 1988: „Von den Gleichnissen“ (Kafka 1920/ 22): Versuch einer Interpretation, MS. Ffm.

Bühl, W.L., 1987: Rezension von N. Luhmann: Ökologische Kommunikation, KZfSS 39: 376-378.

Haferkamp, H./Schmid, H. (Hrsg.) 1987: Sinn, Kommunikation und soziale Differenzierung, Ffm: stw.

Janouch, G. 1981: Gespräche mit Kafka - Aufzeichnungen und Erinnerungen. Ffm: S. Fischer.
Kafka, F. 1961 (Hrsg.: Wagenbach, K.): Die Erzählungen. Ffm: S. Fischer.

Kafka, F. 1986 (Hrsg.: Brod, M.): Hochzeitsvorbereitungen auf dem Lande, Ffm: $\mathrm{S}$. Fischer.

Luhmann, N. 1970: Soziologische Aufklärung 1. Opladen: Westdeutscher Verlag.

Luhmann, N. 1981a: Soziologische Aufklärung 3. Soziales System, Gesellschaft, Organisation. Opladen: Westdeutscher Verlag.

Luhmann, N. 1981b: Gesellschaftsstruktur und Semantik Bd. 2, Ffm: Suhrkamp.

Luhmann, N. 1982: Liebe als Passion: zur Codierung von Intimität, Ffm: Suhrkamp

Luhmann, N. 1986: Ökologische Kommunikation: kann die moderne Gesellschaft sich auf ökologische Gefährdungen einstellen? Opladen: Westdeutscher Verlag.

Luhmann, N. 1987a (Hrsg.: D. Baecker und G. Stanitzek): Archimedes und wir. Interviews. Berlin: Merve.

Luhmann, N. 1987b: Die Richtigkeit soziologischer Theorie: Merkur 41: 36-49.

Luhmann, N. 1987c: Soziologische Aufklärung 4. Beiträge zur funktionalen Differenzierung der Gesellschaft. Opladen: Westdeutscher Verlag.

Luhmann, N. 1988 (1984): Soziale Systeme: Grundriß einer allgemeinen Theorie, Ffm: stw.

Luhmann, N. 1990: Die Wissenschaft der Gesellschaft, Ffm: Suhrkamp.

Politzer, H. 1978: Franz Kafka: Der Künstler. Ffm: Suhrkamp. 